\title{
FUZZY CONTROL OF DIFFERENTIAL DRIVE MOBILE ROBOT FOR MOVING TARGET TRACKING \\ UDC (681.58:004.89):681.5.01
}

\author{
Emina Petrović, Miloš Simonović, Vlastimir Nikolić \\ University of Niš, Faculty of Mechanical Engineering, Niš, Republic of Serbia
}

\begin{abstract}
Tracking of moving objects, including humans has important role in mobile robotics. In this paper, the hierarchical control structure for target/human tracking consisted of high and low level control was presented. The low level subsystem deals with the control of the linear and angular velocities using multivariable PD controller whose parameters are obtained by Particle swarm optimization. The position control of the mobile robot represents the high level control, where we use two fuzzy logic Mamdani controllers for distance and angle control. In order to test the effectiveness of the proposed control scheme a simulation was performed. Two cases, when the mobile robot pursues a target moving along a circular path and when the mobile robot pursues a target moving along a rectangle path, were simulated.
\end{abstract}

Key words: differential drive mobile robot, control, fuzzy logic, moving target tracking

\section{INTRODUCTION}

Tracking a human being with a mobile robot has become essential with modern service robots, from which are expected to work with people and share workspace with them [1] [2] [3]. These robots must be able to detect the position of human in space and monitor it. This capability offers the possibility to use the robots in various applications such as service robotics where robots interact with human providing continuous assistance.

Tracking of moving objects, including humans is one of the most important tasks for autonomous mobile robots. It is defined as the ability of the robot to adjust its position and orientation in accordance with the movement of the moving object in order to reduce the tracking error [4]. In previous years, different control schemes for navigation and tracking with mobile robot were developed.

Methods of artificial intelligence can be applied to various problems encountered with the mobile robotics [4]. These methods are often used because of their simplicity, and

Received December 2, 2016

Corresponding author: Emina Petrović

University of Niš, Faculty of Mechanical Engineering, Aleksandra Medvedeva 14, 18000 Niš, Republic of Serbia

E-mail: emina@masfak.ni.ac.rs 
because they are based on human reasoning and heuristic knowledge and do not require a mathematical model. Several controllers based on fuzzy logic [5] [6], neural networks [7][8] as well as genetic algorithms [9] [10] are developed.

In this paper, the block diagram of proposed hierarchical control scheme for target/human tracking by the nonholonomic mobile robot is shown in Figure 1. Stereo vision module provides the input signal to the control system as the position of the human in $x y$ plane. The distance and angle of target/human are inputs in the high level control where two fuzzy logic Mamdani controllers have been designed: distance controller and angle controller. The control system of the low level is used to make sure that the robot follows the input reference speed and it is consisted of a multivariable PD speed controller.

The paper is organized as follows. In Section 2, the differential drive mobile robot kinematics and dynamics are presented. The fuzzy controller for position control is presented in Section 3. Low level control system is discussed in Section 4. The simulation results of the proposed control algorithm are presented in Section 5.

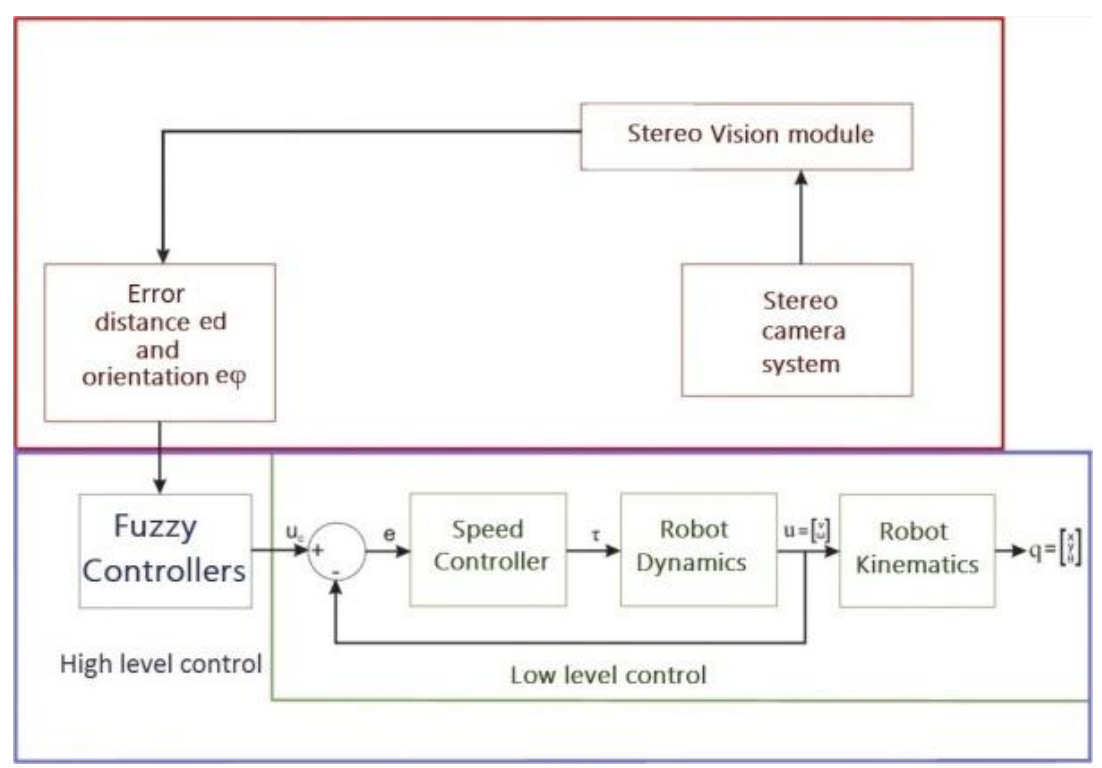

Fig. 1 The control system with two-levels controls

\section{NONHOLONOMIC MOBILE RoBOT DyNAMICS AND KinEMATICS}

Modeling of a differential drive mobile robot consists of kinematic modeling, dynamic modeling and actuator modeling [11]. Kinematic modeling does not consider the forces that affect the motion, but only geometric relationships that govern the system. Dynamic modeling includes affecting forces in the study of the motion. The relationship between the control signal and the input in mechanical system is described by actuator modeling. 
The mobile robot shown in Figure 1 is a typical example of a nonholonomic mechanical system. This mobile robot consists of two wheels mounted on the same axis, with radius $r$, placed at a distance $r_{c}$ from the robot center I, and a free front wheel. Two independent DC motors that provide the necessary torques to the wheels archive the motion and the orientation of the robot.

The center of mass of the platform is assumed to be located at a distance $d$ from the center of the driving wheels axis. The robot platform has a total mass $m$ and a moment of inertia $I_{c}$ around its center of mass. The position of the robot in global inertial reference system $\left(\mathrm{O}, \mathrm{X}_{\mathrm{G}}, \mathrm{Y}_{G}\right)$, is determined by the vector $\mathbf{q}=\left[\begin{array}{lll}x_{I} & y_{I} & \theta\end{array}\right]^{T}$ where $x_{\mathrm{I}}, y_{\mathrm{I}}$ are the coordinates of the center of the driving wheels axis, and $\theta$ is the heading angle of the robot. The reference frame fixed to the mobile robot is $\left(I, X_{R}, Y_{R}\right)$.

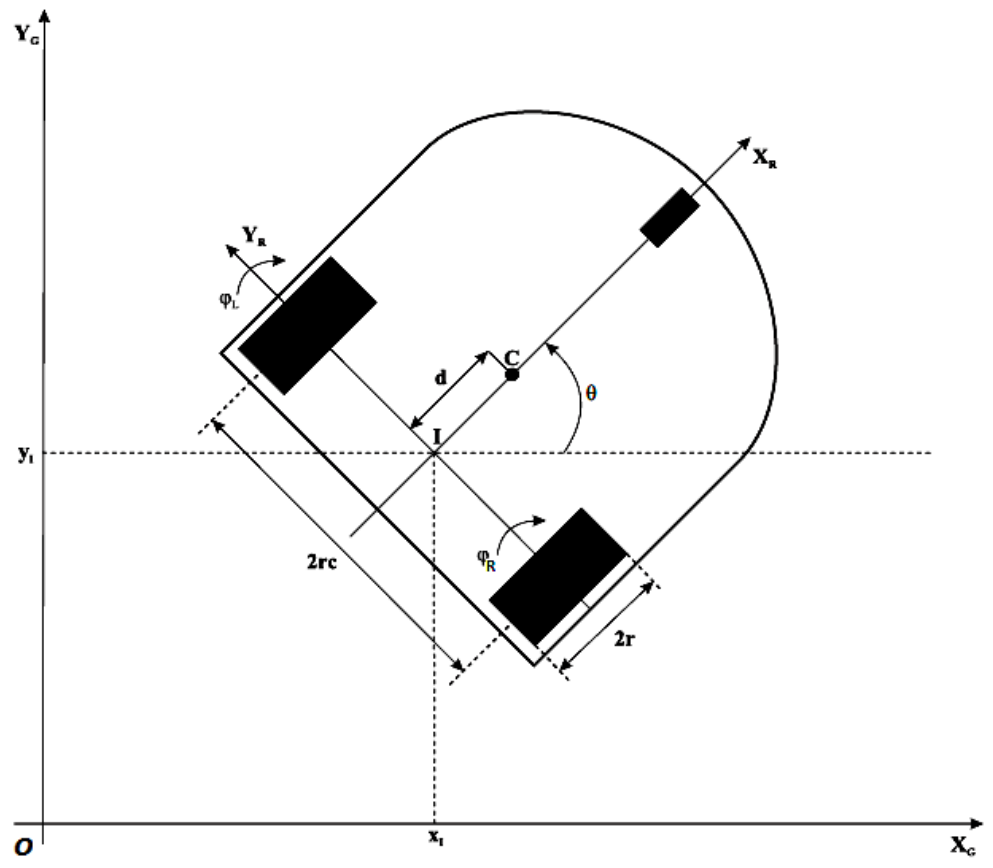

Fig. 2 A differential drive nonholonomic mobile robot

The translation velocity $v$ and angular velocity $\omega$ of the robotic platform are defined as:

$$
\begin{gathered}
v=r \frac{\dot{\varphi}_{R}+\dot{\varphi}_{L}}{2} \\
\omega=\frac{r}{2 r_{c}}\left(\dot{\varphi}_{R}-\dot{\varphi}_{L}\right)
\end{gathered}
$$


We can represent these equations in a matrix, where the velocities are in the inertial frame:

$$
\dot{\boldsymbol{q}}=\left[\begin{array}{c}
\dot{x} \\
\dot{y} \\
\dot{\theta}
\end{array}\right]=\left[\begin{array}{c}
r \frac{\dot{\varphi}_{R}+\dot{\varphi}_{L}}{2} \cos (\theta) \\
r \frac{\dot{\varphi}_{R}+\dot{\varphi}_{L}}{2} \sin (\theta) \\
\frac{r}{2 \mathrm{r}_{\mathrm{c}}}\left(\dot{\varphi}_{R}-\dot{\varphi}_{L}\right)
\end{array}\right]\left[\begin{array}{c}
\dot{\varphi}_{R} \\
\dot{\varphi}_{L}
\end{array}\right]
$$

The equation (3) represents the forward kinematic model of the differential drive mobile robot. Another alternative form for the kinematic model can be obtained by representing the velocities in terms of the linear and angular velocities of a mobile robot.

$$
\dot{\boldsymbol{q}}=\left[\begin{array}{c}
\dot{x} \\
\dot{y} \\
\dot{\theta}
\end{array}\right]=\left[\begin{array}{cc}
\cos \theta & 0 \\
\sin \theta & 0 \\
0 & 1
\end{array}\right]\left[\begin{array}{c}
v \\
\omega
\end{array}\right]
$$

The motion of a differential-drive mobile robot is characterized by nonholonomic constraint equations, i.e. the mobile base satisfies the conditions of pure wheel rolling and no lateral slipping [12].

No lateral slip motion means that the robot can move only in a curved motion (forward and backward) but not sideward [11].

The no lateral slipping constraint is given by:

$$
-\dot{x} \sin \theta+\dot{y} \cos \theta=0
$$

In the robot frame, this condition means that the velocity of the center-point $I$ is zero along the lateral axis.

The pure rolling constraint is described by two equations:

$$
\begin{aligned}
& \dot{x} \cos \theta+\dot{y} \sin \theta+r_{c} \dot{\theta}=r \dot{\varphi}_{R} \\
& \dot{x} \cos \theta+\dot{y} \sin \theta-r_{c} \dot{\theta}=r \dot{\varphi}_{L}
\end{aligned}
$$

This constraint represents the fact that the driving wheels do not slip.

The energy-based Lagrangian approach can be used to derive the dynamic model of the mobile robot, which is represented in the following general form [12]:

$$
M(q) \ddot{q}+C(q, \dot{q}) \dot{q}+F(\dot{q})+G(q)+\tau_{d}=B(q) \tau-A^{T}(q) \lambda
$$

where: $M(q)$ is $n x n$ symmetric positive definite inertia matrix, $C(q, \dot{q})$ is the centripetal and Coriolis matrix, $F(\dot{q})$ is the surface friction matrix, $G(q)$ is the gravitational vector, $\tau_{d}$ is the vector of bounded unknown disturbances including unstructured unmodeled dynamics, $B(q)$ is the input matrix, $\tau$ is the input vector, $A^{T}$ (q) is the matrix associated with the kinematic constraints, and $\lambda$ is the Lagrange multipliers vector. 
For the differential drive mobile robot, the generalized coordinates are selected as:

$$
\dot{q}=\left[\begin{array}{lll}
\dot{x} & \dot{y} & \dot{\theta}
\end{array}\right]^{T}
$$

The robot planar motion leads to the elimination of the gravity terms in the dynamic equation $G(q)=0$, also with the assumption of having negligible friction in the system we can have also $F(\dot{q})=0$.

Next, by expressing the generalized coordinates velocities using the forward kinematic model (4). Then we have

$$
\dot{q}=\left[\begin{array}{c}
\dot{x} \\
\dot{y} \\
\dot{\theta}
\end{array}\right]=S(q) u(t)=\left[\begin{array}{cc}
\cos \theta & 0 \\
\sin \theta & 0 \\
0 & 1
\end{array}\right]\left[\begin{array}{c}
v \\
\omega
\end{array}\right]
$$

This representation is a more proper for control and simulation purposes.

It can be verified that the transformation matrix $S(q)$ has the following relation with the matrix constraint $A(q)$.

$$
S^{T}(q) A^{T}(q)=0
$$

The above equation is useful to eliminate the constraint term from the main dynamic equation.

The time derivative of equation (11) gives

$$
\ddot{q}=\dot{S}(q) u(t)+S(q) \dot{u}(t)
$$

Substituting equations (10) and (12) in the equation (8) we obtain the reduced dynamic model:

$$
\bar{M}(q) \dot{u}(t)+\bar{C}(q, \dot{q}) u(t)+\bar{\tau}_{d}=\bar{B}(q) \tau
$$

where

$$
\begin{gathered}
\bar{M}(q)=\left[\begin{array}{ll}
m & 0 \\
0 & I
\end{array}\right], \bar{C}(q, \dot{q})=\left[\begin{array}{cc}
0 & -m d \dot{\theta} \\
m d \dot{\theta} & 0
\end{array}\right] \\
\bar{B}(q)=\frac{1}{r}\left[\begin{array}{cc}
1 & 1 \\
r_{c} & -r_{c}
\end{array}\right], \tau=\left[\begin{array}{l}
\tau_{R} \\
\tau_{L}
\end{array}\right]
\end{gathered}
$$

\section{FUZZY LOGIC CONTROLLER}

A fuzzy logic controller is a control strategy that uses a fuzzy inference system. It uses heuristic knowledge based on human experts to develop action for mobile robots to achieve different tasks (target tracking, obstacle avoidance) [5],[10]. Also, in real world application utilization of fuzzy logic controllers' methodology is very helpful because it deals with uncertainties.

We define the distance and angle errors variables to mathematically formulate the control objective as follows: 


$$
\begin{gathered}
e_{d}=\sqrt{x_{\text {human }}^{2}+y_{\text {human }}^{2}}-d_{d} \\
e_{\varphi}=\tan ^{-1} \frac{y_{\text {human }}}{x_{\text {human }}}-\varphi_{d}
\end{gathered}
$$

where $x_{\text {human }}$ and $y_{\text {human }}$ present the position of the human in robotic coordinate system obtained from stereo vision system as it shown in Figure 3.

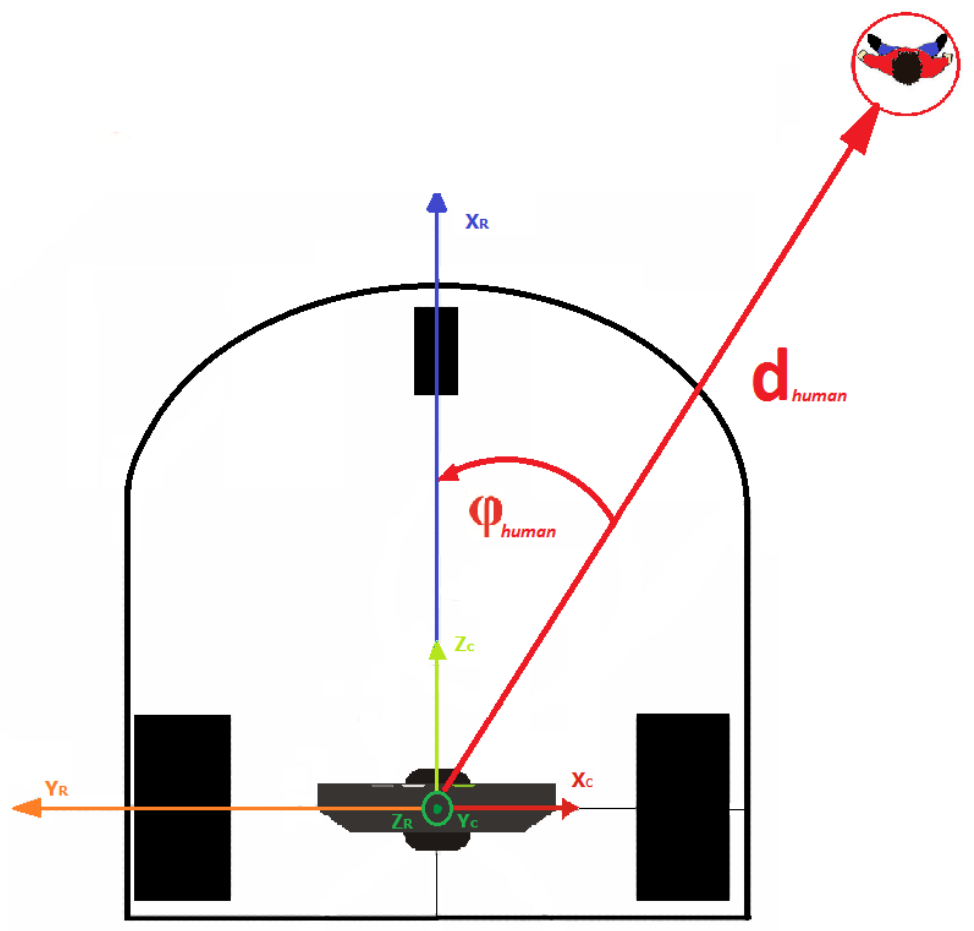

Fig. 3 The distance and angle errors

It is clear that to achieve the control objective, it is sufficient to make the tracking errors tend to be zero.

The fuzzy control rules are represented as a mapping from input linguistic variables $e_{d}$ and $e_{\varphi}$ to output linguistic variables $v$ (linear velocity of mobile robot) and $\omega$ (angular velocity):

$$
\begin{gathered}
v=F L C_{d i s \tan c e}\left(e_{d}\right) \\
\omega=F L C_{\text {angle }}\left(e_{\varphi}\right)
\end{gathered}
$$

The membership functions of input linguistic variable $e_{d}$ and output linguistic variable $v$ are shown on Figure 4. 


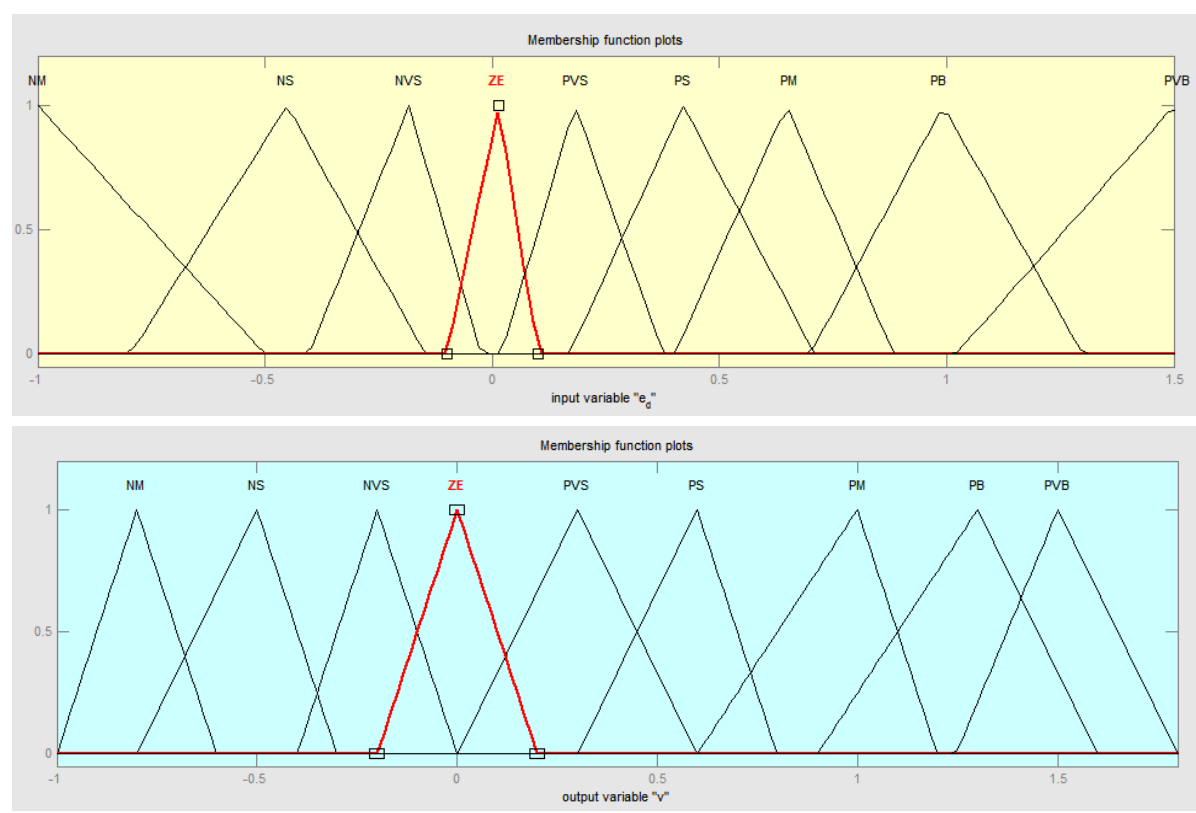

Fig. 4 Membership function of input $e_{d}$ and output $v$

The membership functions of input linguistic variable $e_{\varphi}$ and output linguistic variable $\omega$ are shown on Figure 5.

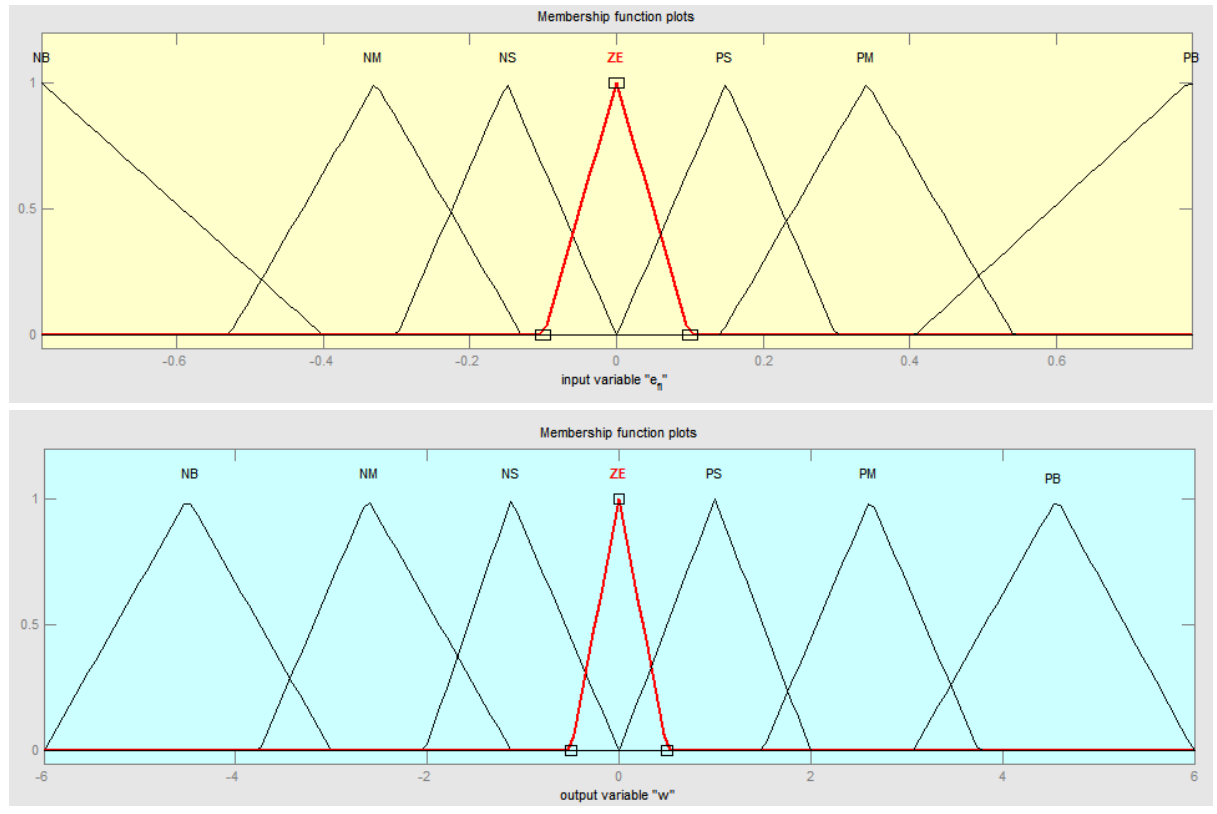

Fig. 5 Membership function of input $e_{\varphi}$ and output $\omega$ 
Table 1 shows the rules' base of implemented fuzzy controllers. For distance controller we have 9 fuzzy rules and for angle controller 7 fuzzy rules.

Table 1 Fuzzy rules

\begin{tabular}{cccc}
\hline \multicolumn{2}{c}{$F L C_{\text {distance }}$} & \multicolumn{2}{c}{$F L C_{\text {angle }}$} \\
\hline$e_{d}$ & $v$ & $e_{\varphi}$ & $\omega$ \\
\hline NB & NB & NB & PB \\
NS & NS & NM & PM \\
VNS & VNS & NS & PS \\
ZE & ZE & ZE & ZE \\
VPS & VPS & PS & NS \\
PS & PS & PM & NM \\
PM & PM & PB & NB \\
PB & PB & - & - \\
VPB & VPB & - & - \\
\hline
\end{tabular}

\section{LOW LEVEL CONTROL SYSTEM - SPEED REGULATOR}

The low level control system consists of a multivariable PD controller for speed regulation. PD controller is responsible for converting the speed at output of the controller in torque for the input to the robot and it is described by the following equations:

$$
\begin{gathered}
\tau=\left[\begin{array}{l}
\tau_{R} \\
\tau_{L}
\end{array}\right]=\bar{B}^{-1}\left[\begin{array}{cc}
g_{1}(t) & 0 \\
0 & g_{2}(t)
\end{array}\right]\left[\begin{array}{l}
e_{v}(t) \\
e_{\omega}(t)
\end{array}\right] \\
g_{1}(t)=K_{p v} e_{v}(t)+K_{d v} \frac{d e_{v}(t)}{d t} \\
g_{2}(t)=K_{p \omega} e_{\omega}(t)+K_{d \omega} S \frac{d e_{\omega}(t)}{d t}
\end{gathered}
$$

where $e_{v}(t)$ is the error of the linear velocity, and $e_{\omega}(t)$ is the error of the angular velocity of mobile robot.

From the obtained simulation results shown in Figure 6, it can be seen that the proposed PD controller successfully tracks the given linear and angular velocity profiles. The values for PD controller parameters are obtained by Particle swarm optimization and they are: $\mathrm{K}_{\mathrm{pv}}=218, \mathrm{~K}_{\mathrm{dv}}=45, \mathrm{~K}_{\mathrm{pw}}=181$ and $\mathrm{K}_{\mathrm{dw}}=28$. 

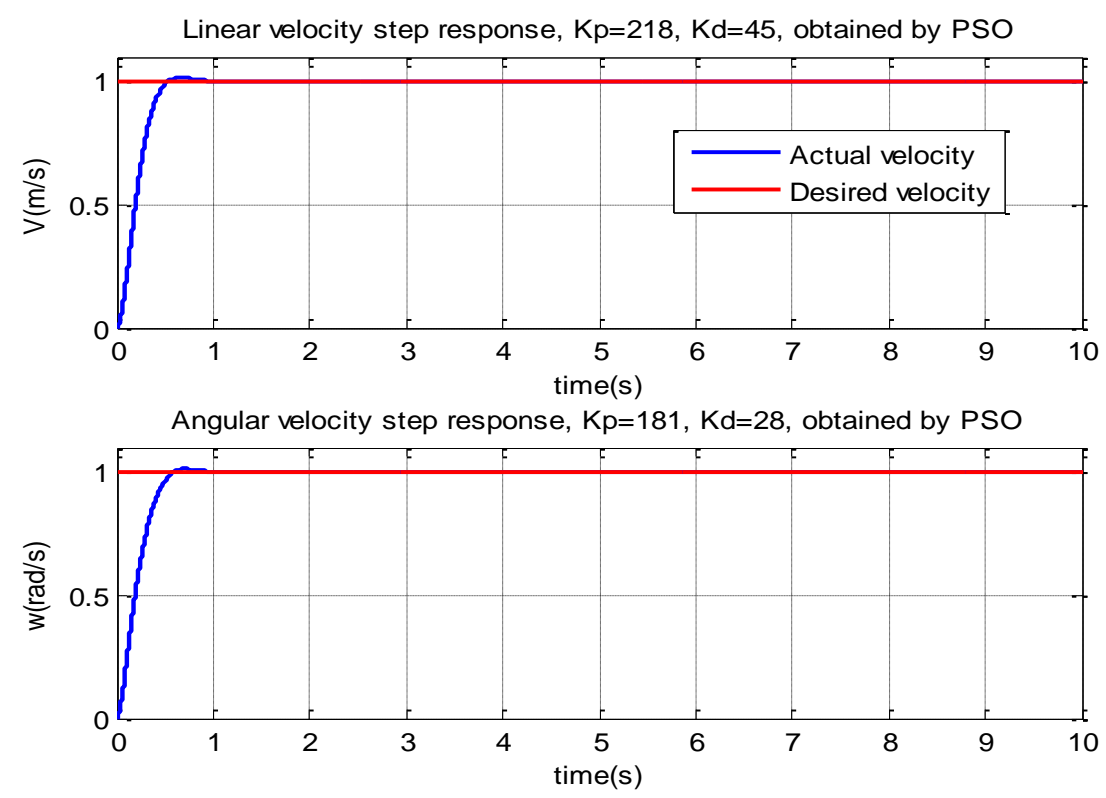

Fig. 6 Step response of linear and angular velocity

\section{SimUlation RESUltS AND CONCLUSION}

In order to test the effectiveness of the proposed control scheme, we have simulated cases when the mobile robot pursues a target moving along a circular path and then a rectangle path. In this simulation, physical parameters and design parameters are $m=3.6$ $\mathrm{kg}, I=0.1 \mathrm{~kg} \cdot \mathrm{m}^{2}, d=0.05, r=0.05, r_{c}=0.2025$.

The target's trajectory in first case can be described by the following equations:

$$
\begin{aligned}
& x_{t}=1+3 \cos t \\
& y_{t}=1+3 \sin t
\end{aligned}
$$

The initial positions of the mobile robot and target are given by $(-0.5,0)$ and $(4,1)$ respectively. The initial orientation of mobile robot is $\theta=0 \mathrm{rad}$. The desired values of the distance and angle from the robot to the target are set to be $d_{d}=0 \mathrm{~m}$ and $\varphi_{d}=0$.

The target's trajectory in the second case is rectangle. The initial positions of the mobile robot and target are given by $(-0.5,1)$ and $(0,0)$ respectively. The initial orientation of mobile robot is $\theta=0 \mathrm{rad}$. The desired values of the distance and angle from the robot to the target are set to be $d_{d}=0 \mathrm{~m}$ and $\varphi_{d}=0$.

The linear velocity of the target in both cases is set to be $1 \mathrm{~m} / \mathrm{s}$, what is an average speed of slow human walk. 


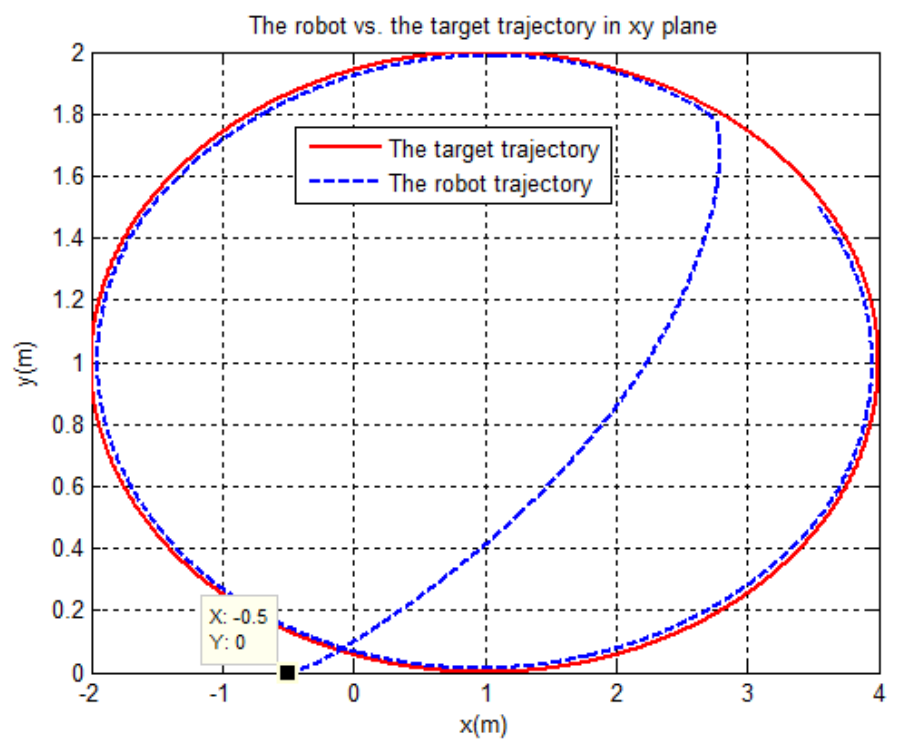

Fig. 7 The target's circular trajectory and robot's trajectory

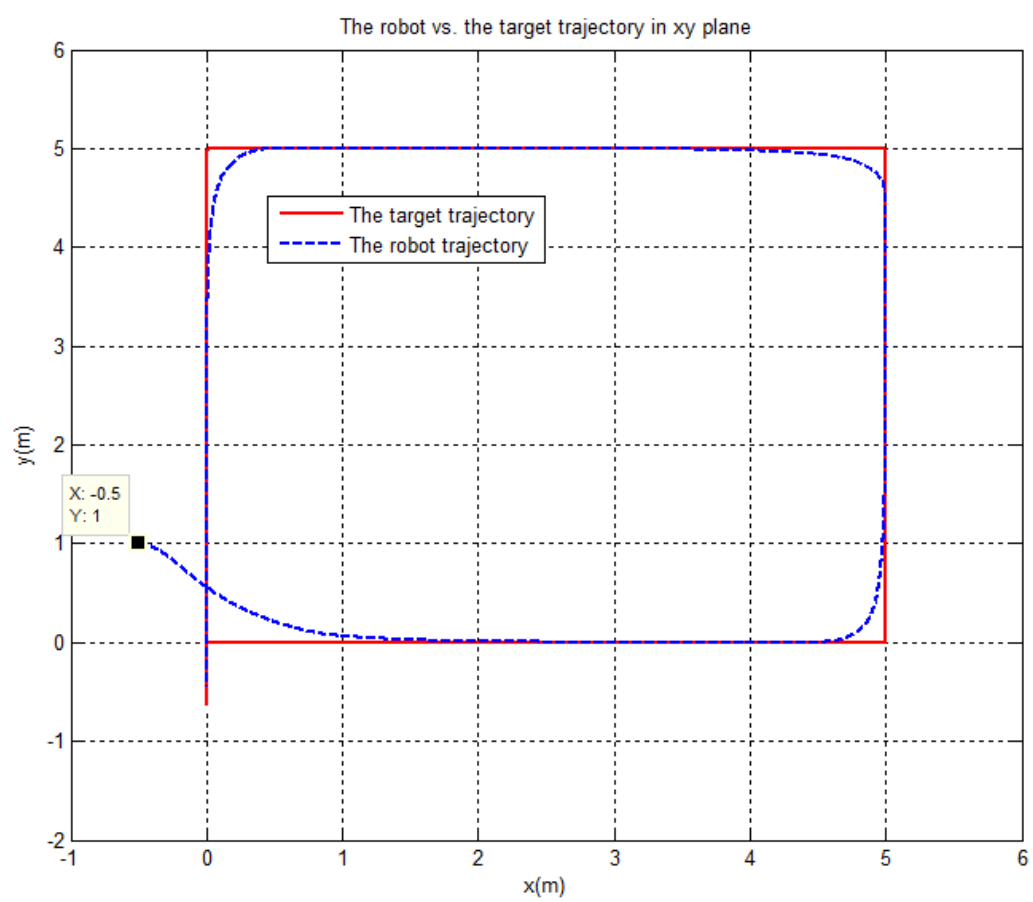

Fig. 8 The target's rectangle trajectory and robot's trajectory 
In this paper, the hierarchical control structure for moving target/human tracking by a mobile robot was presented. The control system is consisted of high and low level control.

The provided simulation results show that the proposed control system successfully track the moving target.

Acknowledgement: This research was supported by the Ministry of Education and Science of Republic of Serbia and DAAD through the project "Robust action recognition for robot-humanrobot synergy".

\section{REFERENCES}

[1] E. Petrović, A. Leu, D. Ristić-Durrant ,V. Nikolić, "Stereo-Vision Based Human Tracking for Robotic Follower", International Journal of Advanced Robotic Systems, vol. 10, 2013.[Online]. Available: http:// dx.doi.org/10.5772/56124.

[2] E. Petrović, Ž. Ćojbašić, D. Ristić-Durrant,V. Nikolić, I. Ćirić, S. Matić, "Kalman filter and Narx neural network for robot vision based human tracking", Facta Universitatis:Series:Automatic Control and Robotics, vol. 12, no.1, pp. 43 - 51, 2013. [Online]. Available: http://casopisi.junis.ni.ac.rs/index.php/FUAutContRob/ article/view/17/5.

[3] D. Ristić-Durrant ,G. Gao, A. Leu, " Low-level sensor fusion-based human tracking for mobile robot", Facta Universitatis: Series:Automatic Control and Robotics, [Online]. Available: http://casopisi.junis.ni.ac.rs/ index.php/FUAutContRob/article/view/1449.

[4] K. Benbouabdallah and Q. D. Zhu, "Improved Genetic Algorithm Lyapunov-Based Controller for Mobile Robot Tracking a Moving Target", Research Journal of Applied Sciences, Engineering and Technology, vol.5, no.15, pp. 4023-4028, 2013. [Online]. Available: http://maxwellsci.com/print/rjaset/v5-40234028.pdf.

[5] T. H. S. Li, S.J. Chang and W. Tong, "Fuzzy target tracking control of autonomous mobile robots by using infrared sensors", IEEE Transaction on Fuzzy System, vol.12, no.4, pp. 491-501, 2004. [Online]. Available: http:// dx.doi.org/ 10.1109/TFUZZ.2004.832526.

[6] S. Blaižić and M. Bernal, "Trajectory Tracking for Nonholonomic Mobile Robots based on Extended Models", Preprints of the 18th IFAC World Congress Milano, Italy, pp. 5938 - 5943, 2011.

[7] O. Mohareri, Mobile robot trajectory tracking using neural network. PhD Thesis, Presented to the faculty of the American University of Sharjah, College of Engineering, , 2009.

[8] Y. Turki, A. Mustafa and I. Hamzah, "Trajectory Tracking Control for Mobile Robot using Wavelet Network", International Journal of Computer Applications, vol. 74, 2013. [Online]. Available: http:// dx.doi.org/ 10.1.1.403.2341

[9] L. Moreno, J. M. Armingol, S. Garrido, A.D.L. Escalera and M. A. Salichs, "Genetic algorithm for mobile robot localization using ultrasonic sensors", Journal of Intelligent and Robotic Systems ,vol.34, no.2 pp. 135-154, 2002. [Online]. Available: http:// dx.doi.org/ 10.1023/A:1015664517164.

[10] K. Benbouabdallah, ,Z. Qi-dan, "Genetic fuzzy logic control technique for a mobile robot tracking a moving target", International Journal of Computer Science Issues, pp. 607-613, 2013. [Online]. Available http://www.ijcsi.org/papers/IJCSI-10-1-1-607-613.pdf.

[11] O. Mohareri, R. Dhaouadi, A. B. Rad, "Indirect adaptive tracking control of a nonholonomic mobile robot via neural networks", Neurocomputing, vol. 88, pp. 54-66, 2012. [Online]. Available: http:// dx.doi.org/ 10.1016/j.neucom.2011.06.035

[12] R. Dhaouadi, A. Abu Hatab, "Dynamic modelling of differential-drive mobile robots using Lagrange and Newton-Euler methodologies: A unified framework", Advances in Robotics and Automation, vol.2, 2013. [Online]. Available: http:// dx.doi.org/ 10.4172/2168-9695.1000107. 